\title{
Mercury seen as never before
}

In its final weeks, the MESSENGER mission reveals fresh details about the planet's scorched surface.

\section{Alexandra Witze}

16 March 2015

\section{THE WOODLANDS, TEXAS}

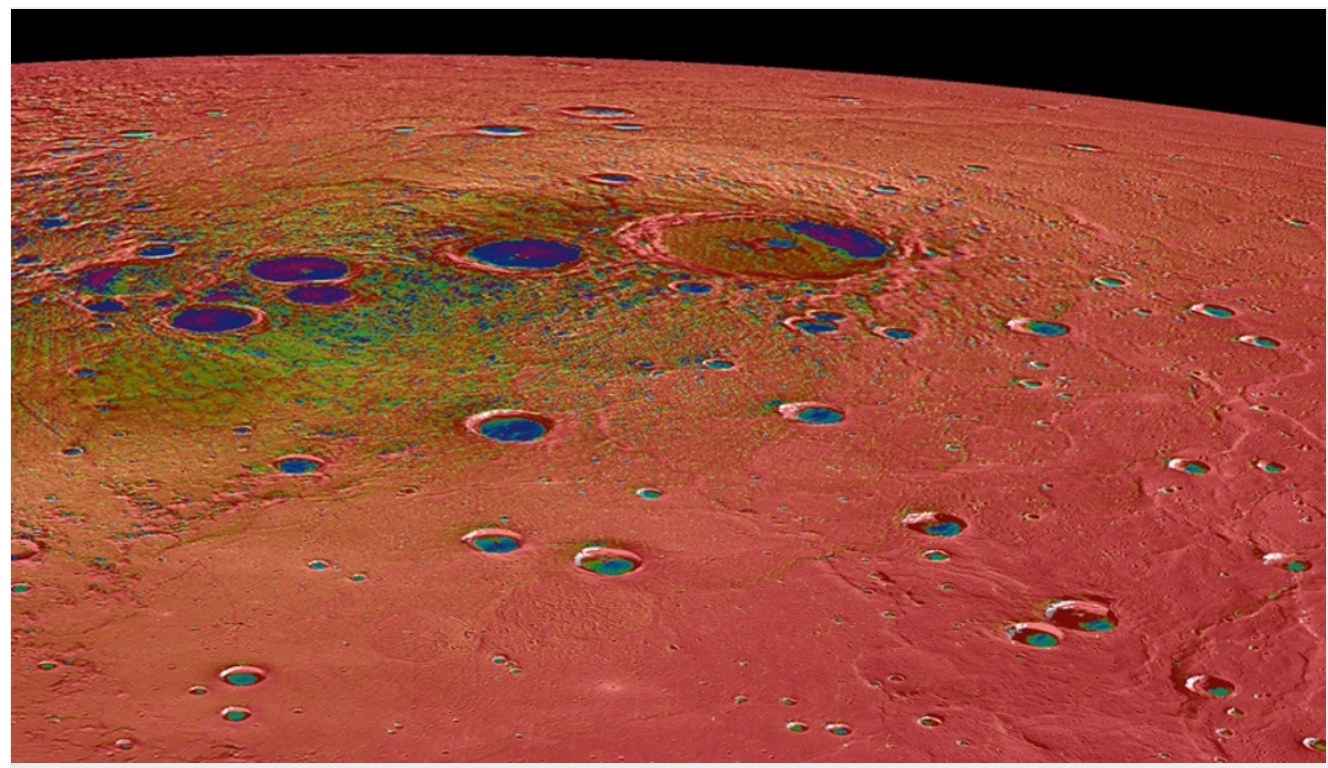

Permanent shadows in craters on Mercury's surface allow ice to survive there.

NASA's MESSENGER spacecraft is set to plunge to its doom on 30 April, ending nearly four years of exploration of Mercury. Before it goes, the mission is sending back the best images of the planet ever taken.

In the shots, released on 16 March, the bottoms of craters reveal ice materializing in pits and swirls, still frozen despite being so close to the Sun. Elsewhere on Mercury, short, staircase-like ridges appear, miniature versions of the huge 'scarps' that the planet is famous for. And tiny hollows mark places where parts of the surface have been scoured away through some kind of powerful space weathering.

MESSENGER, which has been orbiting Mercury since 2011, has nearly run out of propellant to guide it. The spacecraft is currently about 15 kilometres above the planet's surface, the closest that it has ever been.

"We're able to see at close range portions of the planet we haven't seen in such detail before," says Sean Solomon, a geophysicist at the Lamont-Doherty Earth Observatory in Palisades, New York, and principal investigator for the mission. Solomon and other team members presented the findings at the Lunar and Planetary Science Conference in The Woodlands, Texas.

These include new perspectives on one of the mission's biggest discoveries so far — the ice that lurks in permanently shadowed craters near Mercury's poles. The ice remains frozen on sun-baked Mercury because it is never in direct sunlight, says Nancy Chabot, a planetary scientist at the Johns Hopkins University Applied Physics Laboratory in Laurel, Maryland.

In the latest low-altitude images, MESSENGER peers inside roughly a dozen craters near Mercury's poles. At first, photographs of the crater floors just looked black, because the bright crater rims oversaturate the images. But by processing the photographs differently, Chabot could see the dim crater floors popping into view. "We're seeing into these regions where the sun never shines on Mercury," she says. 


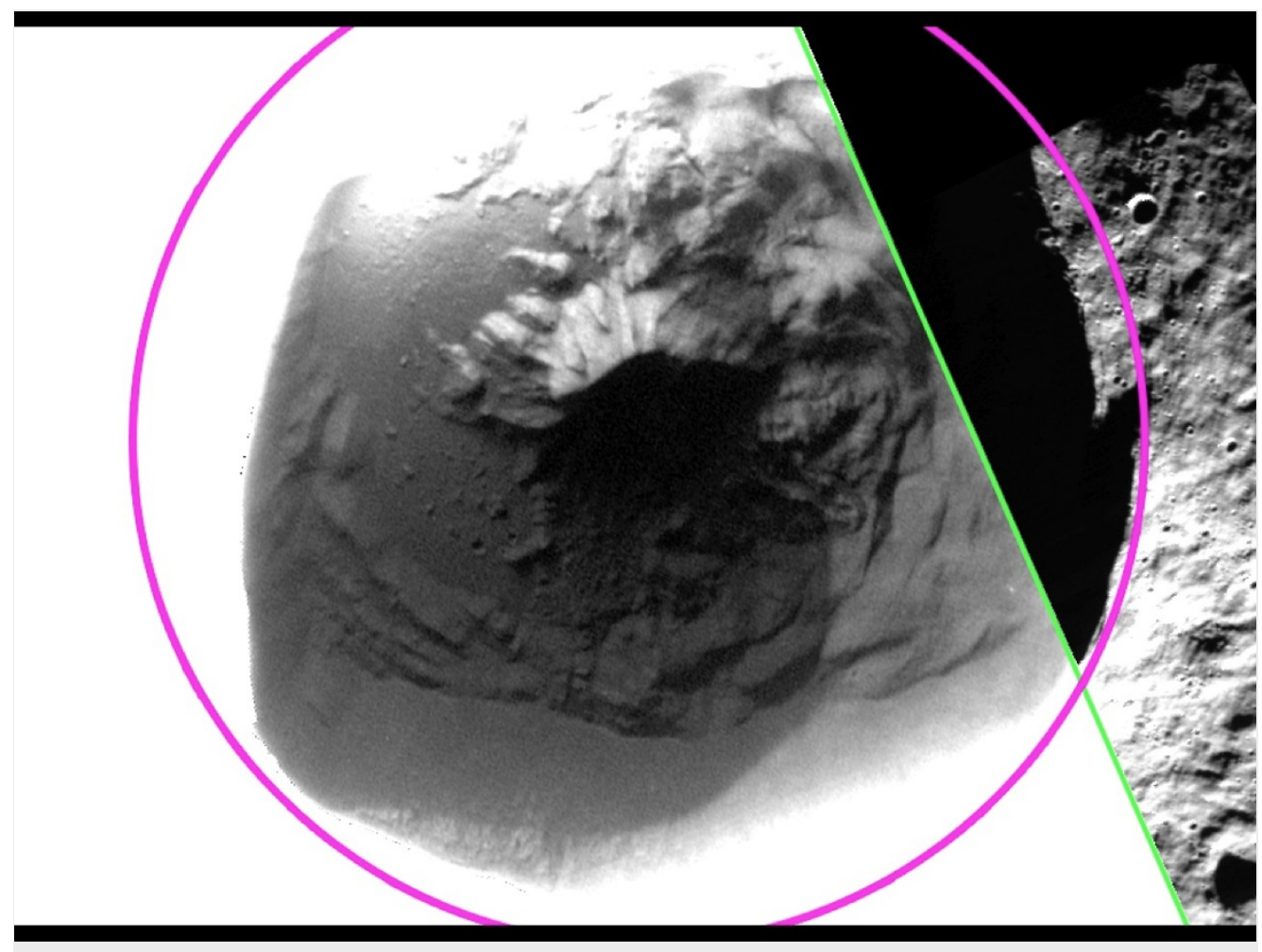

NASA/Camegie/JHUAPL

Mercury's Fuller crater may contain ice from a cosmic collision.

One crater, named Fuller after the architect and visionary Richard Buckminster Fuller, shows patterned areas of light and dark, which might be a result of some sort of dark, carbon-rich material overlying the ice beneath. The boundary between light and dark areas is sharply defined, suggesting that they formed relatively recently. One possibility is that a space rock travelling from farther out in the Solar System slammed into Mercury, depositing water in the form of ice with darker material atop it.

Mercury is also famous for the long ridges, or scarps, that run across much of its surface. The biggest scarps, which can be hundreds of kilometres long, are probably cracks that formed as the planet cooled and shrank over time. Now, MESSENGER has spotted miniature versions of these scarps.

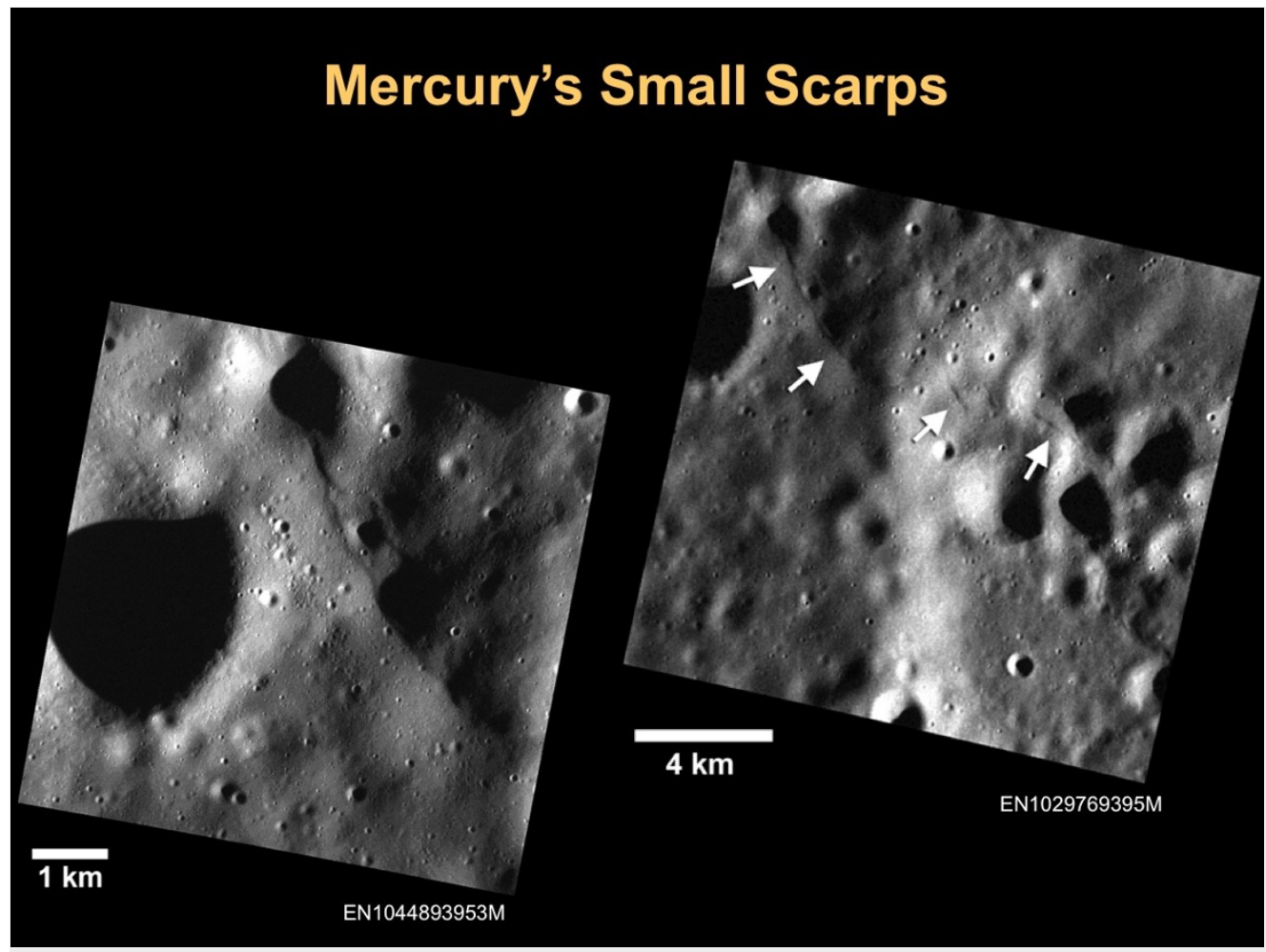

NASA/Camegie/JHAPL 
Long ridges, or 'scarps', run across Mercury's surface. Now researchers have found smaller versions of these formations on the planet.

They seem to occur in clusters, says Thomas Watters, a planetary scientist at the National Air and Space Museum in Washington DC. The small scarps also sometimes occur near collapsed portions of Mercury's crust, which look like portions of Earth's crust that get moved around in active earthquake zones. "These scarps are exciting," says Watters. "These faults are so young that they are probably forming today."

Finally, MESSENGER has also seen details in the mysterious 'hollows' first spotted when the spacecraft went into orbit. Strange, bright areas inside some craters turned out to be irregularly shaped depressions, says David Blewett, a planetary scientist at the Laurel laboratory.

\section{Hollows - Extremely Young}

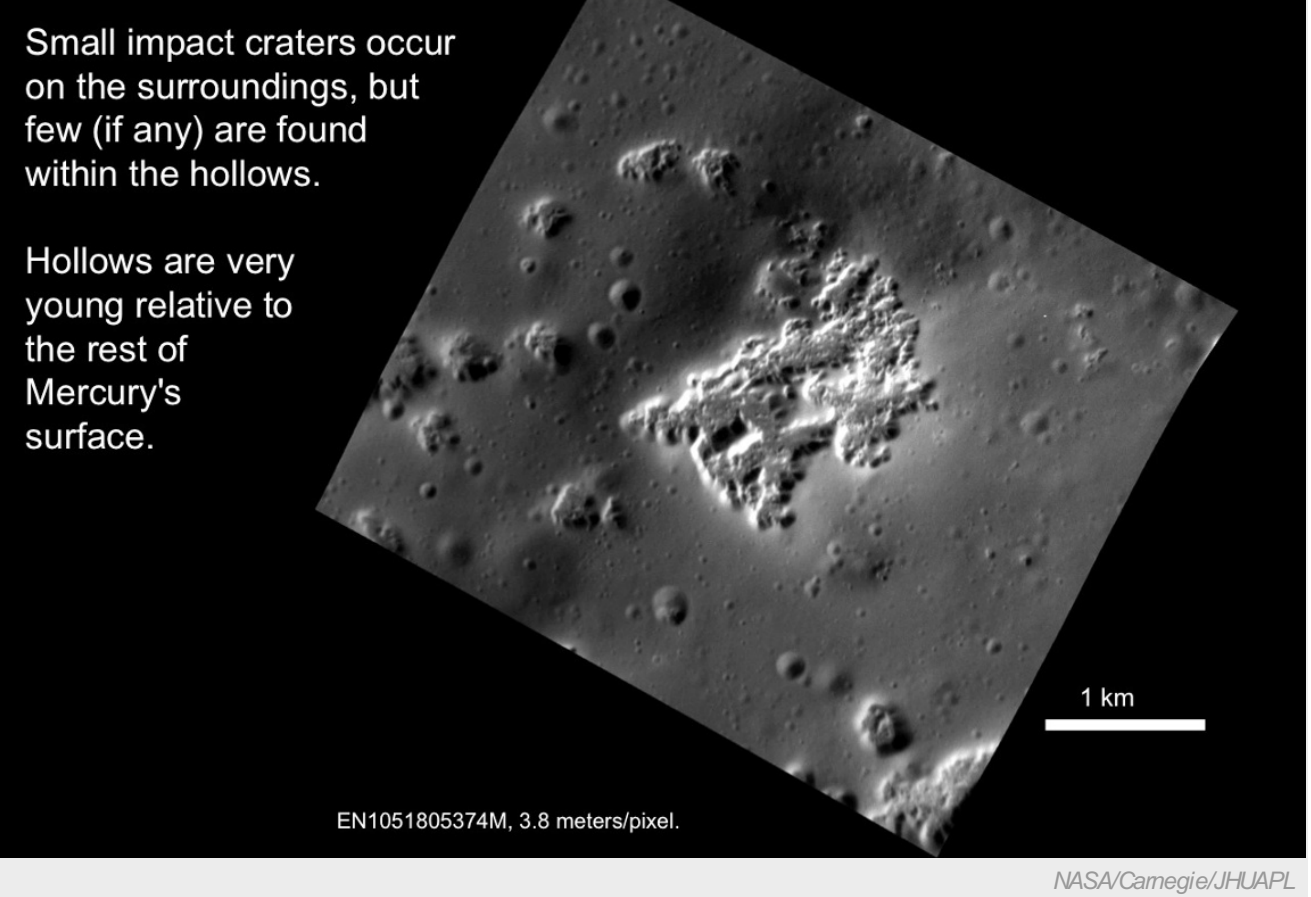

MESSENGER first spotted 'hollows' on Mercury in 2011, but the latest images show these depressions in greater detail.

The close-up images show that the hollows look younger than nearly anything else on Mercury. That also suggests that the planet has been going through some kind of recent changes.

NASA's Dawn spacecraft, which has just arrived at the water-rich asteroid Ceres, is beginning to see intriguing bright glints in crater bottoms. Researchers are anxious to see whether these turn out to be like anything on Mercury.

Mission controllers will perform five more short engine burns to boost MESSENGER's altitude, before the planet's gravity drags it to its inevitable doom. A European Space Agency mission, BepiColombo, is due to arrive at Mercury in 2024, to pick up where MESSENGER leaves off. 